\title{
A Motion Adaptable Needle Placement Instrument Based on Tumor Specific Ultrasonic Image Segmentation
}

\author{
Jae-Sung Hong ${ }^{1}$, Takeyoshi Dohi ${ }^{2}$, Makoto Hasizume ${ }^{3}$, \\ Kozo Konishi ${ }^{3}$, and Nobuhiko Hata ${ }^{2}$ \\ 1 Environmental Studies, Graduate School of Frontier Sciences, \\ The University of Tokyo, 7-3-1 Hongo, Bunkyo-ku, Tokyo 113-8654, Japan \\ hjsshep@atre.t.u-tokyo.ac.jp \\ 2 Mechano-informatics, Graduate School of Information Science and Technology, \\ The University of Tokyo, 7-3-1 Hongo, Bunkyo-ku, Tokyo 113-8654, Japan \\ \{dohi@miki.pe,noby@atre.t\}.u-tokyo.ac.jp \\ 3 Disaster and Emergency Medical, Graduate School of Medical Sciences, \\ Kyushu University, Maidashi 3-1-1, Higashi-ku, Fukuoka, 812-8582, Japan \\ \{konizou@surg2, mhashi@dem\}.med.kyushu-u.ac.jp
}

\begin{abstract}
This paper suggests an ultrasound guided needle insertion instrument which can track target motion in real-time. Under traditional ultrasound guided needle insertion therapies, surgeons have had much burden to find out the precise targeting position, particularly when the organ is moving due to the respiration or heartbeat. We developed a new needle insertion instrument which can track moving target based on visual servo control. In addition, this paper proposed a tumor specific active contour model which can conduct a fast and robust segmentation for tumor, and utilized Hough transform for needle recognition. In the experiment, the proposed system could track a moving phantom successfully at speed of 3 frames/sec processing.
\end{abstract}

\section{Introduction}

Interventional radiology (IR) has been widely accepted in that the therapy is performed with minimal invasiveness so that the period of hospital treatment is significantly reduced [1. In IR therapy, percutaneous microwave coagulation therapy (PMCT) or percutaneous ethanol injection therapy (PEIT) is performed under ultrasonic image-guided environment without performing laparotomy [2]. However these type of operations require highly experienced techniques due to the limitation of visibility on the paths of needle insertion. This imposes a burden on the operator, thus leading to surgical complications such as a missing the target or inaccurate insertion which can cause hemorrhage.

Recently a number of researches on needle accessing instruments based on medical image processing were reported to be in process. Loser et al. proposed a prototypical robot for image-guided interventions optimized for usage inside

T. Dohi and R. Kikinis (Eds.): MICCAI 2002, LNCS 2488, pp. 122-129 2002.

(C) Springer-Verlag Berlin Heidelberg 2002 
a CT gantry [3]. Patriciu et al. proposed that the simple and precise targeting method under portable x-ray fluoroscopy [4], and the authors of this paper developed a manipulator to insert needle for brain tumor using CT images [5]. Nakamura et al. presented a surgical instrument which can track the motion of heartbeat. It can track the target in real-time based on efficient master-slave system, yet this work is performed under CCD camera, i.e. requiring abdominal operation [6].

Inspired by previous works 3456/7 on visual-servoing in medical robotics, this paper introduces a novel method to drive a needle based on real-time ultrasonic image segmentation. The proposed method can compensate for a motion or unexpected trembling of the target caused by respiration or heartbeat and execute a reliable tumor specific segmentation on ultrasonic images to support real-time motion tracking. The image-guided visual servo configuration which is robust to calibration was utilized to achieve real-time tracking for the moving target, and by joining manipulator to ultrasound probe as one unit, eye in hand mechanical organization which is relatively stable to calibration was implemented. In the following sections, we will describe the details of tumor specific ultrasonic image segmentation method, and report about the tracking process with experimental results.

\section{The Robot's Design}

The proposed instrument comprises a five DOF passive arm which is to guide the manipulator to the desired position on the skin providing sufficient visibility and a two DOF active manipulator which is for the control of the access direction of the needle and the distance to the target. Currently we are processing in 2-D space on ultrasonic image. Although an approaching in 3-D space was considered, it turned out to be anatomically impossible because ultrasound probe has to be placed between the ribs in parallel for needle insertion inside liver in most cases. Therefore we assumed the movement of target can be observed on the 2-D image plane. For convenience, we would name the proposed instrument UMI which stands for Ultrasound guided Motion tracking Instrument in the rest part of this paper.

To describe the structure of UMI, the main feature of UMI is that the probe and needle are joined as one unit to be put on the same plane so that the needle position can be observed on the ultrasonic image always during insertion therapy, and this relationship is maintained in spite of any shock or alteration from outside. The needle was inserted by frictional power by motorized rollers of which structure enable the instrument to be made small-sized and the insertion length of needle to be freely adjustable. Inevitable slip of needle can be controlled according to visual feedback. The needle moves around describing imaginary circular arcs and the center of circle corresponds to the puncture point of patient's skin which is fixed as fulcrum of needle. This arc revolution is realized by moving along arc shape guide rail. The fixed guide rail assures the stillness of fulcrum so that the needle does not hurt around skin puncture point. This kind of phys- 


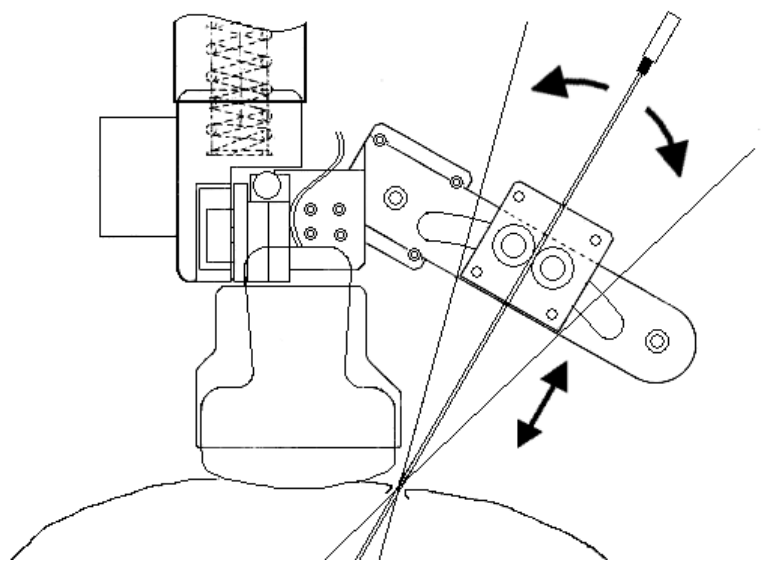

Fig. 1. The probe mounted instrument

ical guide rail often has demerits in burdensome size but UMI has one in small size because only \pm 15 degree give us enough space to move on high resolution ultrasonic images. Fig. 1 shows the front view of the proposed instrument UMI.

\section{Real-Time Tracking Based on Visual Servo Control}

The human body has inevitable internal movements due to respiration, heartbeat or unexpected patient action caused by needle insertion pain. Surgeons often perform needle insertion during the 15 seconds when the patient's respiration is stopped intentionally. Accordingly they have much burden to perform the precise insertion watching the ultrasonic image within a definite time limit, and this kind of therapy needs highly trained technique. UMI can assist the operator to perform accurately and without hastening based on image-guided tracking.

In robotic system, vision has been widely used due to its non-contact sensing and superior informative ability. In medical robotics, the visual servoing has been utilized in the field of minimally invasive surgery to control motion of a camera or medical instrument 89. In most cases, the calibration problem is cumbersome, which is even more difficult in the case of accessing a moving objective in the unstable human body. As compared to position-based image servoing, imagebased visual servo control uses vision alone to stabilize the mechanism as shown in Fig. 2, therefore it is less sensitive to camera calibration 10. In addition, the proposed manipulator uses the eye-in-hand configuration which has a constant relationship between the pose of the camera (probe) and that of the end-effector (needle) [1].

\section{Image Segmentation for Region of Interest}

In ultrasonic images, Regions of interest(ROIs) of our system are tumor and needle. The distance between tumor and needle, plus the status of collision be- 


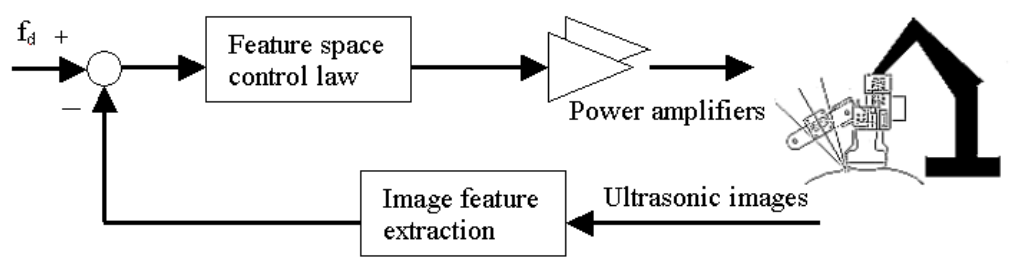

Fig. 2. Image-based visual servoing configuration

tween them are checked from image processing during the therapy. The specified techniques fit for ultrasonic images were utilized.

\subsection{Target Recognition}

The automatic segmentation of ultrasonic image is considerably difficult when using the traditional segmentation methods. Furthermore tumor is more vague than vessel because of its histology. The gradient operators such as sobel or canny detect only some parts of the tumor edge and extracts at once many undesirable edges irrelevant to the tumor.

Kass et al. presented the active contour model (snakes) which can yield a fully connected edge using not only image information but model's own feature [12]. The method defines energy function $E_{\text {snake }}$ which consists of $E_{\text {internal }}$ and $E_{\text {external }}$. Given that there are $N$ control points and $\left(x_{i}, y_{i}\right)$ means the coordinate of the pixel of $i$ th control point, active contour is expressed as follows,

$$
E_{\text {snakes }}=\int_{C} E_{\text {internal }}+E_{\text {external }} d s \cong C\left(x_{i}, y_{i}, \ldots, x_{N}, y_{N}\right)+\sum_{i}^{N} G\left(x_{i}, y_{i}\right)
$$

$C\left(x_{i}, y_{i}\right)$ denotes elastic energy and $G\left(x_{i}, y_{i}\right)$ means gradient of image in our system. Moving the contour to the direction to which the total energy decrease, the optimal contour is determined through the iteration process. This model is robust to the texture of image, so it was also employed as the edge detector for the CT or MRI images 13. In addition because the contour is determined by control points, the contour can be altered easily by changing the position of the control points, that is, according to the processing for only the pixels around control points not the whole pixels in image plane or window, the edge moves and settles down at the final position. This operation is useful for realtime processing and the moving picture segmentation which needs reference to previous edge trail. However, active contour requires the designation of initial contour, and improper designation leads the contour astray or lengthens the time to search right path, therefore considerate inspection is required for initial contour setting.

We devised a method which can set an initial contour most closely to the final contour by using the partially extracted edge produced by a gradient operator and the anatomical prior knowledge. Unlike blood vessels, tumors have elliptical 
shapes in general [14. Accordingly we presumed that the initial contour should be a circle that surrounds the tumor. The radius and coordinate of the center point of the circle can be calculated by referring to the partially extracted edge before, and a small intended enlargement of the calculated radius makes the fittest initial contour which encloses the tumor region. To extract partial, but connected edge of a tumor the edge following method [15] modified from the Delp's algorithm [16], was utilized. Fig. 3] shows the process of contour variation towards the optimal position. We can see the connected contour is extracted successfully which is virtually impossible by traditional edge detectors.

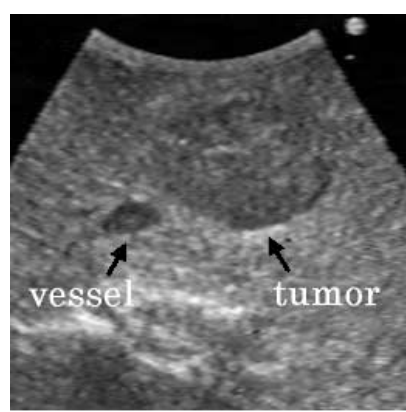

(a)

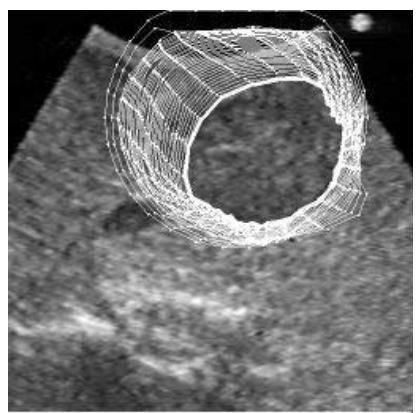

(b)

Fig. 3. Segmentation by Snakes. (a) Original image, (b) Approaching process for tumor contour

\subsection{Needle Recognition}

The needle equipped in UMI is a $20 G$ PTC needle. It usually appears as a straight line on image, but there can be gaps in the line and also some line fragments of other objects irrelative to needle. Therefore, we first have to find out dominant line element on image which represents PTC needle, then locate the endpoint of needle referring to that line.

Hough transform [17 is useful in determination of line parameters such as slope and offset of line even though there are some gaps in line. According to the Hough transform, the pixels on the identical line in image gather into one point in transformed parameter space and the most concentrated point in parameter space is related to a dominant line in image plane which eventually stands for PTC needle. The gradient edge image is used as input for Hough transform. On the other hand in order to locate the endpoint of the needle, the previously acquired line is used as a guide line. Moving along the guide line the connection of edge is verified. When the unconnected point is detected we consider it as endpoint of needle. The small gaps less than a defined threshold are ignored during the check process. Fig. 4 shows the needle on ultrasonic image and the detected endpoint of the needle. 


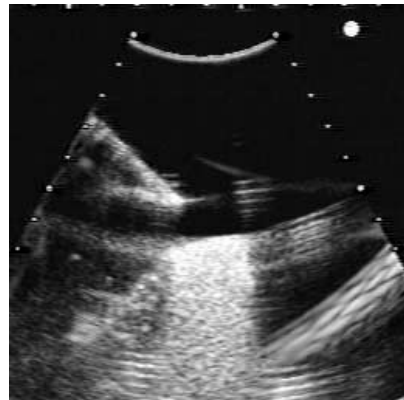

(a)

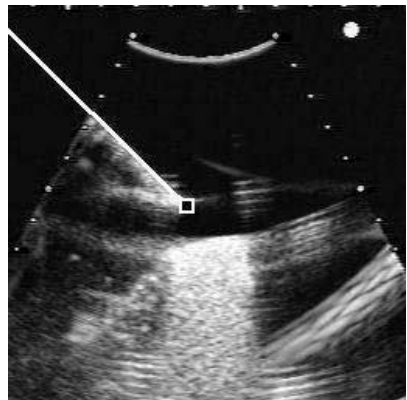

(b)

Fig. 4. Needle recognition using Hough transform, (a) original needle image, (b) detected endpoint of needle

\section{Experiment}

A tumor specific segmentation is tested with ultrasonic images taken under microwave coagulation therapy for liver cancer. On the other hand the tracking ability of the proposed instrument is tested using a phantom. A phantom made by spongy was used as a target. The PC used for image processing and visual servo control is equipped with a Pentium 3 processor, a frame grabber, and a motor control board. The software is programmed using $\mathrm{C}++$ and Tcl/Tk language on a Linux platform. The ultrasound instrument used for this experiment is a $11 \mathrm{MHz}$ probe system. The phantom in a cistern was moved manually and the ultrasonic image was taken during the motion. The end-effector of the actuator was instructed to follow the center of the phantom in real-time. Fig. 5 shows the proposed instrument UMI.

\section{Results and Discussion}

The proposed algorithm could extract the connective contour of the tumor successfully as described previously while the traditional gradient operators in most cases fail to extract. The result images are presented in the previous chapter 4 (See the Fig. 3.) We can also see that the extracted contour is slightly different from the original tumor contour as expected because the computerized algorithm could not performed successfully at the place where the image is severely obscure.

In phantom test, UMI could process 3 frames per a second. Assuming target is moving with speed about $5 \mathrm{~cm} / \mathrm{sec}$, the error distance between target and needle is about $1.7 \mathrm{~cm}$ theoretically. We believe the error caused by time latency would decrease with the improvement of hardware such as use of Pentium 4 processor and software such as thread programming. In the future we will carry out an experiment with ultrasonic liver images using UMI and the results of which will be reported. 


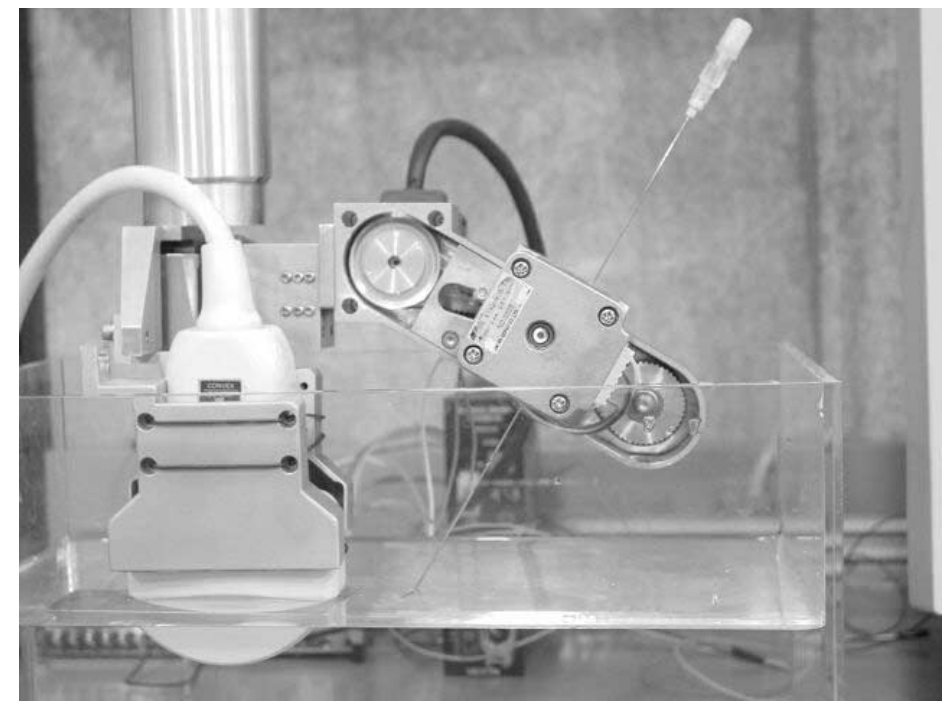

Fig. 5. UMI(Ultrasound guided Motion tracking Instrument)

\section{Conclusion}

This paper suggested an needle insertion instrument which can compensate the unpredictable motion of organs due to the patient respiration or sudden action during the therapy. The tumor specific active contour model based on anatomical knowledge was proposed to achieve the reliable and fast segmentation for relatively vague ultrasonic images. The proposed instrument used visual servo control to track the moving target in real-time, and it was designed as the probe mounted end-effector which ensures always the constant relationship between probe and needle on image. In the experiment, The fine contour for tumor was acquired and the tracking was accomplished for the moving phantom with a 3 frames/sec processing speed.

\section{Acknowledgements}

This work was supported by the Grant-in-Aid for Scientific Research (B13558103, A14702070) in Japan and NH was supported by Toyota Phsical \& Chemical Research Institute, Suzuken Memorial Foundation, Kurata Grants. 


\section{References}

1. M. Kudo: Percutaneous needle placement into hepatic tumors under ultrasound vascular image guidance. Journal of clinical surgery, Vol. 55. (2000) 1551-1555

2. M. Hiroda, T. Becpoo, S. Shimada, M. ogawa: Percutaneous ultrasound-guided microwave coagulation therapy for hepatic neoplasms. Journal of clinical surgery, Vol. 55. (2000) 1557-1560

3. M.H. Loser, N. Navab : A New Robotic System for visually Controlled Percutaneous Interventions under CT Fluoroscopy. MICCAI 2000, Lecture Notes in Computer Science, Vol. 1935. Springer-Verlag (2000) 887-896

4. A. Patriciu, D. Stoianovici, L.L. Whitcomb, T Jarrett, D. Mazilu, A. Stanimir, I. Iordachita, J. Anderson, R. Taylor, L.R. Kavoussi : Motion-Based Robotic Instrument Targeting Under C-Arm Fluoroscopy. MICCAI 2000, Lecture Notes in Computer Science, Vol. 1935. Springer-Verlag (2000) 988-998

5. T. Dohi, "Medical Imaging for Computer Aided Surgery: IEICE Trans. Inf. and Syst., Vol. J83-D-II. (2000) 27-33

6. Y. Nakamura, K. Kishi, H. Kawakami : Heartbeat Sysncronization for Robotic Cardiac Surgery. ICRA2001, IEEE International Conference on Robotics and Automation (2001) 2014-2019

7. S.E. Salcudean, G. Bell, S. Bachmann, et al. : Robot-assisted diagnostic ultrasound - design and feasibility experiments. Proc. MICCAI 1999, Lecture Notes in Computer Science, Vol.1679 (1999) 1062-1071

8. R. H. Taylor, J. Funda, B. Eldridge, D. Larose, M. Talamini, L. Kavoussi, J. Anderson : A telerobotic assistant for laparoscopic surgery. in Proc. Int. Conf. On Advanced Robots (1995) 33-36

9. G.Q. Wei, K. Arbter, G. Hirzinger : Real-Time Visual Servoing for Laparoscopic Surgery. IEEE Eng. in Med. and Biol, Vol.16 (1997) 40-45

10. G.D. Hager, S. Hutchinson and P. Corke: A Tutorial on Visual Servo Control. IEEE Transactions on Robotics and Automation, Vol. 12 (1996) 651-670

11. R. Kelly, R. Carelli, O. Nasis, B. Kuchen, F. Reyes:Stable Visual Servoing of Camera-in-Hand Robotic Systems. IEEE Trans. Mechatoronics, Vol.5 (2000) 39-48

12. M. Kass, A.Witkin, and D. Terzopoulos : Snakes:Active Contour Models. Proceeding of First International Conference on Computer Vision (1987) 259-256

13. M. Mignotte, J.Meunier:A multiscale optimization approach for the dynamic contour-based boundary detection issue. Computerized Medical Imaging and Graphics, Vol 25. (2001) 265-275

14. J.S. Hong, T. Kaneko, R. Sekiguchi and K.H. Park: Automatic Liver Tumor Detection from CT. IEICE Trans. Inf. and Syst., Vol. E84-D. (2001) 741-748

15. C. Choi, S.M. Lee, N.C. Kim, and H. Son: Image segmentation and coding using edge tracing. J. KITE, Vol. 26. (1989) 105-112

16. E.J. Delp and C.H. Chu : Detecting edge segments. IEEE Trans. On Sys. Man and Cybern., Vol SMC-15 (1985) 144-152

17. Hough P.V.C.:A Method and Means for Recognizing Complex Patterns. U.S. Patent No. 3069654 (1962) 